\section{IMP3 is a promising biomarker in urological cancers}

Very few biomarkers are clinically useful for predicting tumor progression in urological cancers. U3 small nucleolar ribonucleoprotein IMP3, is involved in RNA trafficking and stabilization; this fetal oncoprotein influences cell growth and migration during embryogenesis. Two new studies have investigated the potential of this protein as a prognostic marker in renal and bladder cancers.

Sitnikova et al. reported a strong correlation between IMP3 expression and aggressive behavior of bladder carcinomas. IMP3 was expressed in 93\% (26/28) of metastatic urothelial carcinomas, compared with $20 \%$ $(42 / 214)$ of superficial urothelial carcinomas. During follow-up, $60 \%$ of the patients with IMP3-positive T1 superficial urothelial carcinomas developed metastases, compared with $0 \%$ of patients with IMP3-negative tumors. The authors concluded that IMP3-positive tumors were more likely than IMP3-negative tumors to become invasive or metastasize.

Jiang and colleagues found IMP3 to be an independent prognostic biomarker in a subgroup of patients with localized papillary and chromophobe renal cell carcinomas. They showed that patients with IMP3-positive carcinomas had a 10-fold greater risk of progression to distant metastasis than patients with IMP3-negative carcinomas (risk ratio 13.45, 95\% Cl 6.00-30.14; $P<0.001$ ).

Both studies indicate that IMP3 can potentially identify patients with a high risk of developing metastases; this information might help identify patients who could benefit from early aggressive treatment.

Original articles Sitnikova L et al. (2008) IMP3 predicts aggressive superficial urothelial carcinoma of the bladder. Clin Cancer Res 14: 1701-1706

Jiang Z et al. (2008) Oncofetal protein IMP3: a novel molecular marker that predicts metastasis of papillary and chromophobe renal cell carcinomas. Cancer [doi:10.1002/ cncr.23484]

\section{Laparoscopic surgery: a safe option even for vulnerable patients}

Laparoscopic procedures are generally considered to provide good results compared with their open equivalents, but most published studies have included only patients with favorable characteristics. However, the indications for laparoscopic surgery are gradually being broadened, and two new studies support the inclusion of especially vulnerable groups of patients - the very young and the elderly.

Pareek and colleagues describe good outcomes for 26 octogenarian patients who underwent laparoscopic treatment of a solid renal mass: partial $(n=3)$ or radical $(n=10)$ nephrectomy, nephroureterectomy $(n=4)$, cryoablation $(n=7)$, radiofrequency ablation $(n=1)$ and unroofing of a renal cyst $(n=1)$. These patients had multiple comorbidities and correspondingly increased surgical risks, but the complication rate was acceptable and operative characteristics were similar to those expected in favorable-risk populations. There were no treatment-related deaths. Of 22 patients who were alive at last follow-up (range 8-84 months), 19 had no evidence of disease.

Neheman et al. compared the outcomes of 17 newborn or small infants who underwent transperitoneal, laparoscopic, urinary tract surgery with those of a historical cohort of 18 infants matched for age, weight and BMI who underwent open procedures. All children weighed $\leq 6 \mathrm{~kg}$. Despite the technical difficulty of performing laparoscopic surgery in such small infants, the groups had similar outcomes. These authors highlight the need for experienced surgeons and anesthesiologists to perform these procedures.

Original articles Pareek G et al. (2008) Laparoscopic and robotic urology: laparoscopic renal surgery in the octogenarian. BJU Int 101: 867-870

Neheman A (2008) Laparoscopic urinary tract surgery in infants weighing $6 \mathrm{~kg}$ or less: perioperative considerations and comparison to open surgery. J Urol 179: 1534-1538 